\title{
Article \\ Child Labor in Family Tobacco Farms in Southern Brazil: Occupational Exposure and Related Health Problems
}

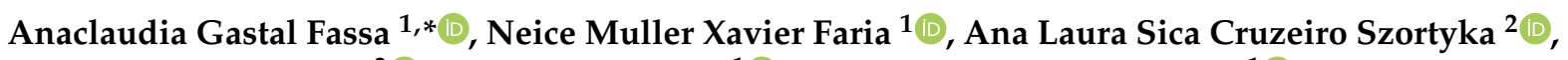 \\ Rodrigo Dalke Meucci ${ }^{3}{ }^{\circledR}$, Nadia Spada Fiori ${ }^{1}\left[{ }^{1}\right.$ and Maitê Peres de Carvalho ${ }^{1}(\mathbb{C}$ \\ 1 Department of Social Medicine, Faculty of Medicine, Federal University of Pelotas, Pelotas 96030-000, Brazil \\ neicef@yahoo.com.br (N.M.X.F.); nsfiori@yahoo.com.br (N.S.F.); maite_carvalho@yahoo.com.br (M.P.d.C.) \\ 2 Psychology Course, Faculty of Medicine, Federal University of Pelotas, Pelotas 96030-000, Brazil; \\ alcruzeiro@gmail.com \\ 3 Faculty of Medicine, Federal University of Rio Grande, Rio Grande 96203-900, Brazil; \\ rodrigodalke@gmail.com \\ * Correspondence: anaclaudia.fassa@gmail.com
}

check for updates

Citation: Fassa, A.G.; Faria, N.M.X.; Szortyka, A.L.S.C.; Meucci, R.D.; Fiori, N.S.; Carvalho, M.P.d. Child Labor in Family Tobacco Farms in Southern Brazil: Occupational Exposure and Related Health Problems. Int. J. Environ. Res. Public Health 2021, 18, 12255. https:// doi.org/10.3390/ijerph182212255

Academic Editor: Paul B. Tchounwou

Received: 18 October 2021

Accepted: 18 November 2021

Published: 22 November 2021

Publisher's Note: MDPI stays neutral with regard to jurisdictional claims in published maps and institutional affiliations.

Copyright: (c) 2021 by the authors. Licensee MDPI, Basel, Switzerland. This article is an open access article distributed under the terms and conditions of the Creative Commons Attribution (CC BY) license (https:/ / creativecommons.org/licenses/by/ $4.0 /)$.

\begin{abstract}
Tobacco farming is considered Hazardous Child Labor in Brazil. This study examined the work of children and adolescents in tobacco farming, characterizing the level of urinary cotinine and the occurrence of Green Tobacco Sickness (GTS), pesticide poisoning, respiratory symptoms, and musculoskeletal disorders. A cross-sectional descriptive study was conducted with a random sample of tobacco growers under 18 years old in Southern Brazil. Ninety-nine young people were interviewed at 79 family farms. The majority began working in agriculture before they were 14 and worked harvesting and tying hands of tobacco; $60 \%$ were 16 or 17 years old, and $51.5 \%$ were male. During their lifetime, $24.5 \%$ reported GTS, and 3\% reported pesticide poisoning. In the previous year, $29.3 \%$ reported low back pain, $6.1 \%$ wheezing, and $16.2 \%$ coughing without having a cold. Half of the 12 young people evaluated had over $100 \mathrm{ng} / \mathrm{mL}$ of urinary cotinine. The study indicates that child laborers do various activities and present a high prevalence of health problems. Health workers should be trained to identify child laborers and their impacts on health. Full-time farm schools could provide knowledge about sustainable agricultural production, reducing the rates of age-grade mismatch, without taking young people away from rural areas.
\end{abstract}

Keywords: child labor; agriculture; rural health; cotinine; tobacco; pesticides; occupational health; epidemiology; cross-sectional studies

\section{Introduction}

Brazil is the world's second largest producer and the largest exporter of tobacco. In the 2019-2020 harvest, the southern region of the country produced more than 600,000 tons of tobacco, involving approximately 146,000 families [1]. Child labor in agriculture and tobacco growing is characterized as hazardous according to Brazilian law [2,3], constituting a violation of human rights, perpetuating the cycle of poverty, contributing to lost educational opportunities, and gender inequality [4-6].

According to the Instituto Brasileiro de Geografia e Estatística (IBGE), in 2019, the occurrence of child labor in Brazil was $4.6 \%$ among children and adolescents aged 5 to 17 years (1.8 million). Of these workers, 53.7\% were 16 and 17 years old, and $66.4 \%$ were male. Among the youngest, aged 5 to $13,16.9 \%$ worked more than $14 \mathrm{~h}$ per week, while those between 16 and 17 years old, 24.2\% worked more than $40 \mathrm{~h}$ per week [7]. In 2016, $47.6 \%$ of workers aged 5 to 13 worked in agriculture [8].

Studies with adults indicate that work in agriculture, especially during the harvest period, requires long working days, working in awkward positions, with repetitive movements and physical exertion [9-13], involving exposure to pesticides, dust, and weather conditions such as heat, hail, frost, and storms [14-16]. In addition to the above, tobacco 
growing also includes exposure to nicotine $[17,18]$. These forms of exposure have been associated with mental health, respiratory and musculoskeletal problems, pesticide poisoning, and green tobacco sickness (GTS) [11-14,16,19-21].

In the context of child labor, a study conducted in Nicaragua found that $21 \%$ of acute pesticide poisoning cases are related to occupational activities, and the work circumstances related to poisonings were similar to those found in adults, such as picking freshly sprayed tobacco leaves, using spraying equipment in bad condition with backpack leakage, preparing pesticide solutions and not using personal protective equipment [22]. In addition, studies on tobacco farming work indicate that age is inversely associated with GTS, since with the prolonged exposure, the workers develop nicotine tolerance $[18,19]$.

There are only a few studies evaluating the respiratory health of children working in agriculture; among them, a study in North Carolina found that $56.4 \%$ of the children had at least one respiratory problem [21]. Within the overall scope of the present study, analyses of adult workers indicate that $21.2 \%$ reported chest pain [12] and $7.4 \%$ reported cervical spine pain [11]. Among adults with chronic low back pain, $37.6 \%$ were unable to carry out some jobs [23]. Doing tasks in awkward positions and carrying heavy loads typical of tobacco growing have been associated with an increase in musculoskeletal disorders [10-12]. Quandt et al. (2021) [13] indicate that $21.3 \%$ of boys working in agriculture had significantly more knee injuries than girls (4.1\%). Children and adolescents may be more vulnerable to some forms of exposure than adults [24,25].

Considering the prevalence of child labor in agriculture, its classification as hazardous child labor, and the scarcity of studies on the subject in Brazil and worldwide, mainly focused on tobacco farming, this study examined the work of children and adolescents in tobacco farming, characterizing the level of urinary cotinine and the occurrence of GTS, pesticide poisoning, respiratory symptoms, and musculoskeletal disorders.

\section{Materials and Methods}

A cross-sectional study was conducted with a random sample of tobacco farmers in the municipality of São Lourenço do Sul, southern Brazil, during the 2011 harvest (January to March). Within this project, 2469 workers were studied; however, only individuals under 18 years old were included in the present article. The southern Brazilian region is responsible for over $95 \%$ of the country's tobacco production; the great majority of the population is of German origin, and its economy is based on tourism and family farming, primarily focused on Virginia tobacco growing [1].

The sample was selected from 3851 invoices provided by the Municipal Treasury Department, referring to tobacco sales and issued in 2009. We estimated about three workers per farm and obtained a simple random sample of 1100 invoices. Tobacco farmers who worked at least fifteen hours per week were eligible for the study. If a selected property was no longer producing tobacco, it was replaced by the nearest tobacco property. Workers residing in the urban area of the municipality or who no longer lived in the municipality were considered ineligible. The selected properties were identified with the help of Community Health Agents from the rural area of São Lourenço do Sul. At the end of the study, 912 properties were identified, and 99 young people from 79 of these properties were interviewed.

Regarding data collection, interviewers specifically trained for the study used PDAs (personal digital assistants) to apply two electronic instruments. One instrument assessed aspects of the farm and was answered by the farm manager, and the other focused on individual aspects, collecting data on sociodemographic and behavioral issues, work activities, workloads, GTS, pesticide poisoning, respiratory symptoms, and musculoskeletal pain. In addition, urinary cotinine samples were collected. Selected questions used to assess health problems can be viewed in the Supplementary Material (File S1).

The schooling variable was categorized according to age-grade mismatch parameters established by the United Nations Children's Fund (UNICEF). The mismatch was calculated in years and represented the gap between the student's age and the recommended age 
for the grade he or she is attending. When the difference between the student's age and expected age for the grade was two years or more, this was considered an "age-grade mismatch" [26]. Workers who smoked one or more cigarettes per day of any type during the last month were considered smokers, while those who reported having quit smoking more than one month ago were considered former smokers. Passive smokers were those who had people close to them who frequently smoked in their presence. In order to characterize alcoholic beverage intake, we asked about consumption on weekdays (Monday through Friday) and at the weekend (Saturdays and Sundays) in the past 30 days, considering a standard dose to be equivalent to about 10-12 $\mathrm{g}$ of pure ethanol, as established by the World Health Organization [27].

The presence of musculoskeletal pain was assessed by the Nordic Musculoskeletal Questionnaire [28], which has been validated in Brazil [29] and has already been used in other studies [30,31]. Each participant was asked if they had had back pain in the 12 months prior to the interview. To those who reported pain, a picture of the human body standing in an upright position was shown, with the cervical, thoracic, and lumbar regions highlighted to identify the site(s) of pain. Pesticide poisoning was self-reported by the workers. GTS during lifetime was defined as the occurrence of dizziness or headache and nausea or vomiting up to two days after handling tobacco [32].

Urinary cotinine was measured in workers with or without symptoms of GTS, who worked on farms located in the two districts with the highest tobacco production in São Lourenço do Sul, and who had applied pesticides in the year before the survey. Cotinine is the primary metabolite of nicotine and the most suitable parameter to assess exposure to tobacco, as it has greater stability and a longer half-life than nicotine [33,34]. Urine samples were stored in a freezer at $-10{ }^{\circ} \mathrm{C}$ for conservation and sent weekly to the Municipal Health Department of São Lourenço do Sul, which would transport the samples to the Toxicology Laboratory of the Pontifical Catholic University of Rio Grande do Sul in the city of Porto Alegre/RS. The High-Performance Liquid Chromatography (HPLC) method with an ultraviolet detector was used, and the urinary cotinine concentration was calculated by its absorbance at $260 \mathrm{~nm}$, considering the peak area and the calibration curve $(\mathrm{r}>0.99)$, using an already standardized technique [35]. With the urinary cotinine among nonsmokers as a reference (lower than $20 \mathrm{ng} / \mathrm{mL}$ ), the concentration was categorized as less than $20 \mathrm{ng} / \mathrm{mL}$, 20-100 ng/mL, and more than $100 \mathrm{ng} / \mathrm{mL}$.

Data analysis was performed using Stata $12.0^{\circledR}$ (StataCorp LLC, College Station, TX, USA). Descriptions of continuous variables were evaluated through their means. The frequency of the categorical variables was verified by the prevalence and confidence intervals $(95 \% \mathrm{CI})$, stratified by sex and age. In order to assess differences by age group and sex for each variable, the Chi-square Test for Heterogeneity was used, and, in the case of small numbers (cells with less than five observations), the Fisher's Exact Test was utilized. Missing data were excluded from the analysis.

This study was approved by the Research Ethics Committee of the Federal University of Pelotas (Protocol Code no. 11/2010). The participants' legal guardians and the young workers themselves were informed about the research topic, the right not to participate or to withdraw from participating at any time, and the confidentiality of individual information. Those who agreed to participate signed the Informed Consent Form.

\section{Results}

The sample was comprised of 99 workers of both sexes, under 18 years old, who worked in 79 family properties. These farms had on average 37.3 hectares $(95 \%$ CI $24.0-$ 50.6), of which 7.5 hectares (95\%CI 5.9-9.1) were used for tobacco growing. The majority of the farm properties $(72.7 \%$ ) had at least five farming machines, and $83.2 \%$ had a tractor. About $93 \%$ of the farmers worked on farms with at least one vehicle (motorcycle, car, pickup truck, or truck). The main pesticides used on these farms were: herbicides (clomazone $72.6 \%$, glyphosate $65.3 \%$, sulfentrazone $48.4 \%$ ), insecticides (neonicotinoids 
$76.8 \%$, organophosphates $63.2 \%$, pyrethroids $38.9 \%$ ), fungicides (dithiocarbamates $65.3 \%$, metalaxyl $49.5 \%$, iprodione $33.7 \%$ ), and growth regulators (flumetralin $92.6 \%$ ).

Among the 99 young people, $60 \%$ were between 16 and 17 years old, $51.5 \%$ were male, around $10 \%$ were smokers or former smokers, and almost $60 \%$ reported being passive smokers. As for alcohol consumption at the weekend, more than $60 \%$ of the 16 and 17 years old, and almost $25 \%$ of the 14 and 15 years old, reported drinking one or more doses daily. During the week, around $13 \%$ of the young respondents reported consuming alcoholic beverages occasionally. The age-school grade mismatch was 3.5\% among 14 and 15 years old, and $44.1 \%$ among 16 and 17 years old. Approximately $80 \%$ of the young people worked on farm properties that produced up to $10,000 \mathrm{~kg}$ of tobacco, with those under 16 years of age concentrated in small and medium-sized farm properties. Almost all respondents had some type of curing barn on their farm property $(98 \%)$, and $28.4 \%$ had an electric curing barn. Passive smoking $(p=0.037)$ and alcohol consumption at the weekend $(p=0.005)$ were higher among male workers. Among the young male workers, $76 \%$ worked at farms producing less than $10,000 \mathrm{~kg}$ of tobacco, and $88.8 \%$ of females also worked at farms of this size $(p=0.043)$ (Table 1$)$.

Among the young people interviewed, $66 \%$ began doing farm work before they were 14 years old, approximately $14 \%$ began working with pesticides at $14-15$ years old and $52.5 \%$ dedicated at least $5 \mathrm{~h}$ per day to farm work outside the harvest period. Among those under 14 and those who were 14 and 15 years old, $36.4 \%$ and $41.3 \%$, respectively, worked more than $4 \mathrm{~h}$ a day doing farming activities outside the harvest period. Among those who were 16 and 17 years old, $39 \%$ worked more than $7 \mathrm{~h}$ a day. During the harvest, $84.6 \%$ of the young people worked more than $4 \mathrm{~h}$ a day, and among those older than $13,65.9 \%$ worked more than $7 \mathrm{~h}$ a day. Approximately $20 \%$ of the young people did not perform domestic work, while $34.3 \%$ and $20.2 \%$, respectively, spent more than $3 \mathrm{~h}$ a day doing domestic work both outside and during the harvest. More than $70 \%$ of those under 14 years old had less than $11 \mathrm{~h}$ of leisure time both during and outside the harvest, while among those who were 14 to 17 years old, $59 \%$ and $48.8 \%$, respectively, had more than $10 \mathrm{~h}$ of leisure time per week, outside the harvest and during the harvest (Table 2).

When stratifying by sex, we found that boys worked more with pesticides $(47.1 \%)$ than girls $(12.5 \%)$. Domestic work for more than $3 \mathrm{~h}$ a day was more frequently carried out by girls, both outside (54.1\%) and during harvest time (33.3\%) (Table 2).

The activities undertaken varied little according to age group. Most young people were involved in tying hands of tobacco $(95 \%)$, harvesting tobacco in the last week $(85.7 \%)$, tending animals $(83.8 \%)$, transplanting tobacco $(82.2 \%)$, lifting tobacco sticks $(78.8 \%)$, and sowing tobacco $(74.7 \%)$. About $60 \%$ were also involved in carrying and transporting green leaves, baling tobacco, harvesting wet leaves, and grading tobacco leaves. More than half of the young people drove tractors or other farm machinery, $37.4 \%$ climbed high into the curing barn, and $23.2 \%$ drove cars or trucks. These activities were mainly performed among 16 and 17 years old and less frequently by those younger than 16 . Controlling the temperature of curing barns during the night was performed by about $10 \%$ of the young people. Among the 14 to 17 years old, $17 \%$ cut trees with a chainsaw, and $15.9 \%$ reported carrying more than $50 \mathrm{~kg}$. Among the 16 and 17 years old, $13.6 \%$ carried bales weighing about $50 \mathrm{~kg}$. More than $80 \%$ of the 14 to 17 years old, and $27.3 \%$ of those under 14 years old, worked in a bending position. Wearing protective clothing during tobacco harvesting was reported by $38.4 \%$ of the individuals interviewed, and wearing gloves for harvesting was mentioned by $49.5 \%$ (Table 3 ).

When stratifying by sex, the following activities were performed more by boys: sowing tobacco $(88.2 \%)$, carrying and transporting green leaves (54.9\%), climbing high into the curing barn (54.9\%), driving tractors/farm machinery (88\%), driving cars/trucks (41.2\%), cutting trees with chainsaw $(30.6 \%)$, delimbing trees $(47.1 \%)$, controlling curing barn temperature during the day $(47.1 \%)$ and at night (17.6\%) and carrying weight $(55 \%)$. Looking after the vegetable garden (54.2\%) was more frequent among girls (Table 3 ). 
Regarding tobacco plantation activities involving exposure to pesticides, we found that children under 14 did not engage in this type of activity. Among 16 and 17 years old, approximately $18 \%$ prepared pesticide solution and applied pesticides, $12.1 \%$ went into the plantation shortly after pesticide application, and $8.6 \%$ cleaned pesticide application equipment. The main activities involving pesticide exposure carried out by 14 and 15 years old included washing clothes worn during pesticide application $(13.8 \%)$ and re-entering the treated field shortly after pesticide application (6.9\%). Preparing pesticide mixture $(p=0.013)$, applying pesticides $(p=0.043)$, and cleaning equipment $(p=0.040)$ were performed more frequently by boys while washing clothes worn during pesticide application was more frequent among girls $(p=0.010)$ (Table 4$)$.

Urinary cotinine was analyzed in 12 of the 99 young people, all of them nonsmokers; $33.3 \%$ had 20 to $100 \mathrm{ng} / \mathrm{mL}$, and $50 \%$ had more than $100 \mathrm{ng} / \mathrm{mL}$ of cotinine in their urine (Table 5).

Approximately $25 \%$ of the interviewees reported an occurrence of GTS once during their lifetime, and $13.3 \%$ reported having had it three times or more. Among workers aged 14 to $17,3.5 \%$, all of whom were male, reported pesticide poisoning during their lifetime (Table 5).

Regarding respiratory symptoms, $16.2 \%$ of the young people reported coughing without having a cold, and $6.1 \%$ reported wheezing in the last 12 months. As for musculoskeletal disorders, low back pain was the most frequent, affecting $29.3 \%$ of the workers and increasing with age, with $39 \%$ frequency among workers aged 16 and 17 . Thoracic spine pain in the last year was reported by $26.3 \%$ of the workers. Neck pain in the last year was reported by $3.0 \%$, all of whom were female (Table 5). Figure 1 shows the prevalence of health problems.

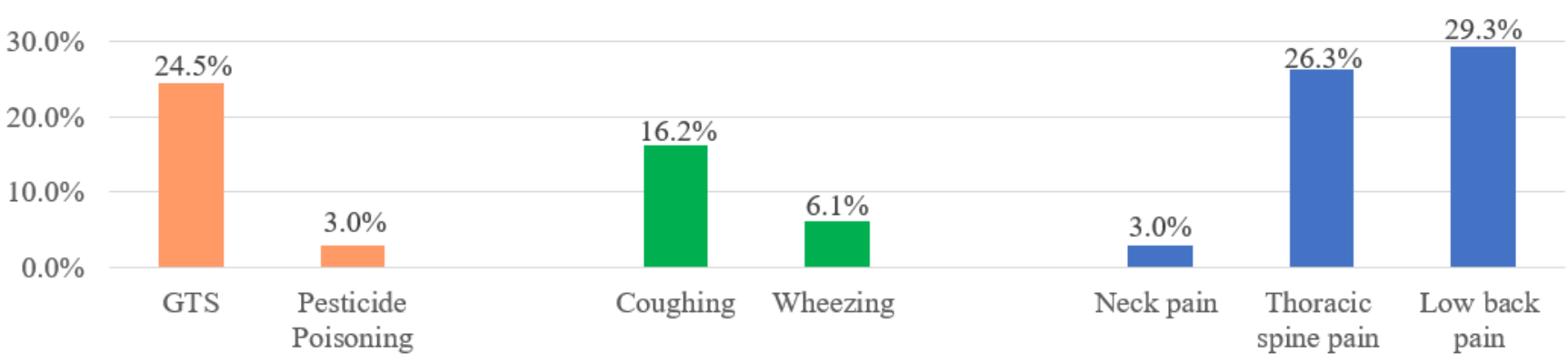

Health Problems

Figure 1. Prevalence of Health Problems among Children Tobacco Growers. São Lourenço do Sul, Rio Grande do Sul, Brazil, 2011 ( $n=99)$. 
Table 1. Demographic, socioeconomic, and behavioral characterization of tobacco farmers stratified by age and sex. São Lourenço do Sul, Rio Grande do Sul, Brazil, 2011. ( $n=99)$.

\begin{tabular}{|c|c|c|c|c|c|c|c|c|}
\hline \multirow{3}{*}{ Variables } & \multirow{3}{*}{$\begin{array}{c}\text { Total }(n=99) \\
\%\end{array}$} & \multicolumn{4}{|c|}{ Age Group } & \multicolumn{3}{|c|}{ Sex } \\
\hline & & $<14$ Years $(n=11)$ & 14-15 Years $(n=29)$ & $16-17$ Years $(n=59)$ & \multirow{2}{*}{$p$-Value for Age } & Male $(n=51)$ & Female $(n=48)$ & \multirow{2}{*}{$p$-Value for Sex } \\
\hline & & $\%$ & $\%$ & $\%$ & & $\%$ & $\%$ & \\
\hline Sex & & & & & $0.975^{\mathrm{a}}$ & & & - \\
\hline Male & 51.5 & 54.5 & 51.7 & 50.8 & & - & - & \\
\hline Female & 48.5 & 45.5 & 48.3 & 49.2 & & - & - & \\
\hline Age-school grade mismatch * & & & & & $<0.001^{\mathrm{b}}$ & & & $0.065^{\mathrm{a}}$ \\
\hline Adequate for age & 72.7 & 100 & 96.5 & 55.9 & & 64.7 & 81.3 & \\
\hline Inadequate for age & 27.3 & 0 & 3.5 & 44.1 & & 35.3 & 18.7 & \\
\hline No & 92.9 & 90.9 & 100 & 89.8 & & 88.2 & 97.9 & \\
\hline Yes & 4.1 & 0 & 0 & 6.8 & & 7.8 & 0 & \\
\hline Former smoker & 3.0 & 9.1 & 0 & 3.4 & & 4.0 & 2.1 & \\
\hline Passive smoking & & & & & $0.913^{\mathrm{b}}$ & & & $0.037^{\mathrm{b}}$ \\
\hline No & 43.4 & 36.4 & 44.8 & 44.1 & & 33.3 & 54.2 & \\
\hline Yes & 56.6 & 63.6 & 55.2 & 55.9 & & 66.7 & 45.8 & \\
\hline $\begin{array}{l}\text { Alcohol consumption at } \\
\text { weekends }\end{array}$ & & & & & $0.007^{b}$ & & & $0.005^{b}$ \\
\hline Has never drunk & 33.3 & 54.5 & 44.9 & 23.7 & & 25.5 & 41.6 & \\
\hline Drinks occasionally & 20.2 & 27.3 & 31.0 & 13.6 & & 21.6 & 18.8 & \\
\hline$<1$ dose/day & 16.1 & 0 & 13.8 & 20.4 & & 13.7 & 18.8 & \\
\hline 3 or more doses/day & 15.2 & 9.1 & 10.3 & 18.6 & & 27.4 & 2.0 & \\
\hline $\begin{array}{l}\text { Alcohol consumption during } \\
\text { the week }\end{array}$ & & & & & $0.977^{\mathrm{b}}$ & & & $0.202^{b}$ \\
\hline Has never drunk & 82.8 & 90.9 & 79.2 & 83.0 & & 76.5 & 89.6 & \\
\hline Drinks occasionally & 13.1 & 9.1 & 13.8 & 13.6 & & 15.7 & 10.4 & \\
\hline$<1$ dose /day & 2.0 & 0 & 3.5 & 1.7 & & 3.9 & 0 & \\
\hline 2 or more doses/day & 2.0 & 0 & 3.5 & 1.7 & & 3.9 & 0 & \\
\hline $\begin{array}{l}\text { Amount of tobacco produced } \\
\qquad(\mathrm{Kg})^{* *}\end{array}$ & & & & & $0.074^{\mathrm{b}}$ & & & $0.043^{\mathrm{b}}$ \\
\hline $1-5000$ & 32.6 & 20.0 & 37.0 & 32.8 & & 22.0 & 44.4 & \\
\hline $5001-10,000$ & 49.5 & 80.0 & 55.6 & 41.3 & & 54.0 & 44.4 & \\
\hline $10,001-36,000$ & 17.9 & 0 & 7.4 & 25.9 & & 24.0 & 11.2 & \\
\hline$\underset{* *}{\text { Curing barn on the property }}$ & & & & & $0.756^{b}$ & & & $0.433^{\mathrm{b}}$ \\
\hline No curing barn & 2.1 & 0 & 3.7 & 1.7 & & 2.0 & 2.2 & \\
\hline Only conventional curing barn & 69.5 & 70.0 & 74.1 & 67.3 & & 64.0 & 75.6 & \\
\hline Only electric curing barn & 10.5 & 0 & 7.4 & 13.8 & & 10.0 & 11.1 & \\
\hline Both & 17.9 & 30.0 & 14.8 & 17.2 & & 24.0 & 11.1 & \\
\hline
\end{tabular}


Table 2. Organization and division of tobacco farmers' work, stratified by age and sex. São Lourenço do Sul, Rio Grande do Sul, Brazil, 2011. $(n=99)$.

\begin{tabular}{|c|c|c|c|c|c|c|c|c|}
\hline \multirow{3}{*}{ Variables } & \multirow{3}{*}{$\begin{array}{c}\text { Total }(n=99) \\
\%\end{array}$} & \multicolumn{4}{|c|}{ Age Group } & \multicolumn{3}{|c|}{ Sex } \\
\hline & & $<14$ Years $(n=11)$ & $14-15$ Years $(n=29)$ & 16-17 Years $(n=59)$ & \multirow{2}{*}{$p$-Value for Age } & Male $(n=51)$ & Female $(n=48)$ & \multirow{2}{*}{$p$-Value for Sex } \\
\hline & & $\%$ & $\%$ & $\%$ & & $\%$ & $\%$ & \\
\hline $\begin{array}{l}\text { Age at which began doing } \\
\text { farm work * }\end{array}$ & & & & & $0.049^{b}$ & & & $0.752^{b}$ \\
\hline$<10$ years & 3.3 & 0 & 3.6 & 3.5 & & 4.2 & 2.3 & \\
\hline $10-11$ years & 23.1 & 83.3 & 14.3 & 21.1 & & 22.9 & 23.3 & \\
\hline $12-13$ years & 39.5 & 16.7 & 53.5 & 35.1 & & 43.7 & 34.9 & \\
\hline $14-15$ years & 33.0 & 0 & 28.6 & 38.5 & & 29.2 & 37.2 & \\
\hline $16-17$ years & 1.1 & 0 & 0 & 1.8 & & 0 & 2.3 & \\
\hline $\begin{array}{l}\text { Age at which began working } \\
\text { with pesticide }\end{array}$ & & & & & $0.132^{\mathrm{b}}$ & & & $0.001^{\mathrm{b}}$ \\
\hline Does not work with pesticide & 69.7 & 81.8 & 72.4 & 66.0 & & 52.9 & 87.5 & \\
\hline$<14$ years & 9.1 & 18.2 & 13.8 & 5.1 & & 11.8 & 6.2 & \\
\hline $14-15$ years & 14.1 & 0 & 13.8 & 17.0 & & 23.5 & 4.2 & \\
\hline $16-17$ years & 7.1 & 0 & 0 & 11.9 & & 11.8 & 2.1 & \\
\hline $\begin{array}{l}\text { Daily hours of farm work } \\
\text { outside harvest period }\end{array}$ & & & & & $0.095^{\mathrm{b}}$ & & & $0.061^{\mathrm{b}}$ \\
\hline Does not work & 10.1 & 0 & 10.3 & 11.9 & & 5.9 & 14.6 & \\
\hline$<5 \mathrm{~h}$ & 37.4 & 63.6 & 48.4 & 27.1 & & 29.4 & 45.8 & \\
\hline $5-7 \mathrm{~h}$ & 23.2 & 27.3 & 24.1 & 22.0 & & 25.5 & 20.8 & \\
\hline $\begin{array}{l}\text { Daily hours of farm work } \\
\text { during the harvest }\end{array}$ & & & & & $0.006^{\mathrm{b}}$ & & & $0.681^{\mathrm{a}}$ \\
\hline$<5 \mathrm{~h}$ & 15.2 & 9.1 & 27.6 & 10.2 & & 11.8 & 18.8 & \\
\hline $5-7 \mathrm{~h}$ & 23.2 & 63.6 & 13.8 & 20.3 & & 23.5 & 22.9 & \\
\hline$>7 \mathrm{~h}$ & 61.6 & 27.3 & 58.6 & 69.5 & & 64.7 & 58.3 & \\
\hline $\begin{array}{l}\text { Daily hours of domestic work } \\
\text { outside harvest period }\end{array}$ & & & & & $0.839^{\mathrm{b}}$ & & & $<0.001^{\mathrm{b}}$ \\
\hline Does not work & 24.2 & 27.3 & 27.6 & 22.0 & & 41.2 & 6.3 & \\
\hline $1-3 \mathrm{~h}$ & 41.4 & 27.3 & 41.4 & 44.1 & & 43.1 & 39.6 & \\
\hline$>3 \mathrm{~h}$ & 34.3 & 45.4 & 31.0 & 33.9 & & 15.7 & 54.1 & \\
\hline $\begin{array}{l}\text { Daily hours of domestic work } \\
\text { during the harvest }\end{array}$ & & & & & $0.908^{b}$ & & & $<0.001^{\mathrm{b}}$ \\
\hline Does not work & 22.2 & 18.2 & 27.6 & 20.3 & & 41.2 & 2.1 & \\
\hline $1-3 \mathrm{~h}$ & 57.6 & 54.5 & 55.2 & 59.4 & & 21.0 & 64.6 & \\
\hline$>3 \mathrm{~h}$ & 20.2 & 27.3 & 17.2 & 20.3 & & 7.8 & 33.3 & \\
\hline
\end{tabular}


Table 2. Cont.

\begin{tabular}{|c|c|c|c|c|c|c|c|c|}
\hline \multirow{3}{*}{ Variables } & \multirow{3}{*}{$\begin{array}{c}\text { Total }(n=99) \\
\%\end{array}$} & \multicolumn{4}{|c|}{ Age Group } & \multicolumn{3}{|c|}{ Sex } \\
\hline & & $<14$ Years $(n=11)$ & 14-15 Years $(n=29)$ & $16-17$ Years $(n=59)$ & \multirow{2}{*}{$p$-Value for Age } & Male $(n=51)$ & Female $(n=48)$ & \multirow{2}{*}{$p$-Value for Sex } \\
\hline & & $\%$ & $\%$ & $\%$ & & $\%$ & $\%$ & \\
\hline $\begin{array}{l}\text { Weekly hours of leisure } \\
\text { outside harvest period }\end{array}$ & & & & & $0.229^{\mathrm{b}}$ & & & $0.619^{b}$ \\
\hline$<6 \mathrm{~h}$ & 11.1 & 27.3 & 6.9 & 10.2 & & 13.7 & 8.3 & \\
\hline $6-10 \mathrm{~h}$ & 33.3 & 45.4 & 31.0 & 32.2 & & 29.4 & 37.5 & \\
\hline$>10 \mathrm{~h}$ & 55.6 & 27.3 & 62.1 & 57.6 & & 56.9 & 54.2 & \\
\hline $\begin{array}{l}\text { Weekly hours of leisure } \\
\text { during the harvest }\end{array}$ & & & & & $0.343^{\mathrm{b}}$ & & & $0.523^{\mathrm{a}}$ \\
\hline$<6 \mathrm{~h}$ & 15.2 & 18.2 & 17.2 & 13.6 & & 13.7 & 16.7 & \\
\hline $6-10 \mathrm{~h}$ & 39.4 & 63.6 & 34.5 & 37.3 & & 35.3 & 43.7 & \\
\hline$>10 \mathrm{~h}$ & 45.4 & 18.2 & 48.3 & 49.1 & & 51.0 & 39.6 & \\
\hline
\end{tabular}

Table 3. Tobacco worker activities and workloads, stratified by age group and sex. São Lourenço do Sul, Rio Grande do Sul, Brazil, 2011. ( $n=99)$.

\begin{tabular}{|c|c|c|c|c|c|c|c|c|}
\hline \multirow{3}{*}{ Variables } & \multirow{3}{*}{$\begin{array}{c}\text { Total }(n=99) \\
\%\end{array}$} & \multicolumn{4}{|c|}{ Age Group } & \multicolumn{3}{|c|}{ Sex } \\
\hline & & $<14$ Years $(n=11)$ & $14-15$ Years $(n=29)$ & $16-17$ Years $(n=59)$ & \multirow{2}{*}{$p$-Value for Age } & Male $(n=51)$ & Female $(n=48)$ & \multirow{2}{*}{$p$-Value for Sex } \\
\hline & & $\%$ & $\%$ & $\%$ & & $\%$ & $\%$ & \\
\hline Sowing tobacco & & & & & $0.436^{\mathrm{b}}$ & & & $0.001^{\mathrm{a}}$ \\
\hline No & 25.3 & 18.2 & 34.5 & 22.0 & & 11.8 & 39.6 & \\
\hline Yes & 74.7 & 81.8 & 65.5 & 78.0 & & 88.2 & 60.4 & \\
\hline Transplanting tobacco & & & & & $0.063^{\mathrm{b}}$ & & & $0.686^{\mathrm{a}}$ \\
\hline No & 17.2 & 27.3 & 27.6 & 10.2 & & 15.7 & 18.7 & \\
\hline Yes & 82.2 & 72.7 & 72.4 & 89.8 & & 84.3 & 81.3 & \\
\hline No & 14.3 & 18.2 & 14.3 & 13.6 & & 14.0 & 14.6 & \\
\hline Yes & 85.7 & 81.8 & 85.7 & 86.4 & & 86.0 & 85.4 & \\
\hline Harvesting wet leaves & & & & & $0.537^{\mathrm{a}}$ & & & $0.287^{a}$ \\
\hline No & 38.4 & 45.5 & 44.8 & 33.9 & & 33.3 & 43.7 & \\
\hline Yes & 61.6 & 54.5 & 55.2 & 66.1 & & 66.7 & 56.3 & \\
\hline $\begin{array}{l}\text { Carrying and transporting } \\
\text { green leaves }\end{array}$ & & & & & $0.227^{b}$ & & & $<0.001^{\mathrm{a}}$ \\
\hline No & 37.4 & 45.5 & 48.3 & 30.5 & & 45.1 & 79.2 & \\
\hline Yes & 62.6 & 54.6 & 51.7 & 69.5 & & 54.9 & 20.8 & \\
\hline
\end{tabular}


Table 3. Cont.

\begin{tabular}{|c|c|c|c|c|c|c|c|c|}
\hline \multirow{3}{*}{ Variables } & \multirow{3}{*}{$\begin{array}{c}\text { Total }(n=99) \\
\%\end{array}$} & \multicolumn{4}{|c|}{ Age Group } & \multicolumn{3}{|c|}{ Sex } \\
\hline & & $<14$ Years $(n=11)$ & $14-15$ Years $(n=29)$ & 16-17 Years $(n=59)$ & \multirow{2}{*}{$p$-Value for Age } & Male $(n=51)$ & Female $(n=48)$ & \multirow{2}{*}{$p$-Value for Sex } \\
\hline & & $\%$ & $\%$ & $\%$ & & $\%$ & $\%$ & \\
\hline $\begin{array}{l}\text { Lifting tobacco sticks (Around } \\
\qquad 12-14 \mathrm{Kg} \text { ) }\end{array}$ & & & & & $0.292^{b}$ & & & $0.127^{\mathrm{a}}$ \\
\hline No & 21.2 & 36.4 & 13.8 & 22.0 & & 54.9 & 39.6 & \\
\hline Yes & 78.8 & 63.6 & 86.2 & 78.0 & & 45.1 & 60.4 & \\
\hline Grading tobacco leaves & & & & & $0.915^{\mathrm{a}}$ & & & $0.510^{\mathrm{a}}$ \\
\hline No & 40.4 & 45.5 & 41.4 & 39.0 & & 37.3 & 43.8 & \\
\hline Yes & 59.6 & 54.5 & 58.6 & 61.0 & & 62.7 & 56.2 & \\
\hline Tying hands of tobacco & & & & & $1.000^{\mathrm{b}}$ & & & $0.363^{\mathrm{b}}$ \\
\hline No & 5.0 & 0 & 3.5 & 6.8 & & 7.8 & 2.1 & \\
\hline Yes & 95.0 & 100 & 96.5 & 93.2 & & 92.2 & 97.9 & \\
\hline Baling tobacco & & & & & $0.196^{\mathrm{a}}$ & & & $<0.001^{\mathrm{a}}$ \\
\hline No & 37.4 & 54.5 & 44.8 & 30.5 & & 19.6 & 56.3 & \\
\hline Yes & 62.6 & 45.5 & 55.2 & 69.5 & & 80.4 & 43.7 & \\
\hline $\begin{array}{c}\text { Transporting tobacco bales } \\
\text { (around } 50 \mathrm{Kg} \text { ) }\end{array}$ & & & & & $0.065^{\mathrm{b}}$ & & & $0.270^{\mathrm{b}}$ \\
\hline No & 91.9 & 100 & 100 & 86.4 & & 88.2 & 95.8 & \\
\hline Yes & 8.1 & 0 & 0 & 13.6 & & 11.8 & 4.2 & \\
\hline $\begin{array}{l}\text { Climbing high into the } \\
\text { curing barn }\end{array}$ & & & & & $0.231^{\mathrm{b}}$ & & & $<0.001^{\mathrm{a}}$ \\
\hline No & 62.6 & 81.8 & 51.7 & 64.4 & & 45.1 & 81.3 & \\
\hline Yes & 37.4 & 18.2 & 48.3 & 35.6 & & 54.9 & 18.7 & \\
\hline $\begin{array}{l}\text { Taking care of the } \\
\text { vegetable garden }\end{array}$ & & & & & $0.051^{\mathrm{a}}$ & & & $0.007^{\mathrm{a}}$ \\
\hline No & 59.6 & 45.5 & 44.8 & 69.5 & & 72.6 & 45.8 & \\
\hline Yes & 40.4 & 54.5 & 55.2 & 30.5 & & 17.4 & 54.2 & \\
\hline Tending animals & & & & & $0.004^{\mathrm{b}}$ & & & $0.076^{\mathrm{b}}$ \\
\hline No & 16.2 & 18.2 & 34.5 & 6.8 & & 9.8 & 22.9 & \\
\hline Yes & 83.8 & 81.8 & 65.5 & 93.2 & & 90.2 & 77.1 & \\
\hline $\begin{array}{l}\text { Driving tractor/ } \\
\text { farm machines * }\end{array}$ & & & & & $0.443^{b}$ & & & $<0.001^{\mathrm{a}}$ \\
\hline No & 46.9 & 63.6 & 48.3 & 43.1 & & 22.0 & 72.9 & \\
\hline Yes & 53.1 & 36.4 & 51.7 & 56.9 & & 88.0 & 27.1 & \\
\hline Driving car/truck & & & & & $0.645^{\mathrm{b}}$ & & & $<0.001^{b}$ \\
\hline No & 76.8 & 81.8 & 82.8 & 72.9 & & 58.8 & 95.8 & \\
\hline Yes & 23.2 & 18.2 & 17.2 & 27.1 & & 41.2 & 4.2 & \\
\hline Cutting trees * & & & & & $0.293^{b}$ & & & $<0.001^{b}$ \\
\hline Does not cut & 78.1 & 100 & 77.8 & 74.1 & & 61.2 & 95.7 & \\
\hline Cut with chainsaw & 15.6 & 0 & 11.1 & 20.7 & & 30.6 & 0 & \\
\hline Cut with other equipment & 6.3 & 0 & 11.1 & 5.2 & & 8.2 & 4.3 & \\
\hline
\end{tabular}


Table 3. Cont.

\begin{tabular}{|c|c|c|c|c|c|c|c|c|}
\hline \multirow{3}{*}{ Variables } & \multirow{3}{*}{$\begin{array}{c}\text { Total }(n=99) \\
\%\end{array}$} & \multicolumn{4}{|c|}{ Age Group } & \multicolumn{3}{|c|}{ Sex } \\
\hline & & $<14$ Years $(n=11)$ & 14-15 Years $(n=29)$ & $16-17$ Years $(n=59)$ & \multirow{2}{*}{$p$-Value for Age } & Male $(n=51)$ & Female $(n=48)$ & \multirow{2}{*}{$p$-Value for Sex } \\
\hline & & $\%$ & $\%$ & $\%$ & & $\%$ & $\%$ & \\
\hline Delimbing trees & & & & & $0.046^{\mathrm{b}}$ & & & $<0.001^{\mathrm{a}}$ \\
\hline No & 68.7 & 100 & 65.5 & 64.4 & & 52.9 & 85.4 & \\
\hline Yes & 31.3 & 0 & 34.5 & 35.6 & & 47.1 & 14.6 & \\
\hline $\begin{array}{l}\text { Controlling the temperature } \\
\text { of the curing barn during } \\
\text { the day }\end{array}$ & & & & & $0.077^{\mathrm{a}}$ & & & $0.023^{a}$ \\
\hline Yes & 36.4 & 54.5 & 20.7 & 40.7 & & 47.1 & 25.0 & \\
\hline $\begin{array}{l}\text { Controlling the temperature } \\
\text { of the curing barn during } \\
\text { the night }\end{array}$ & & & & & $0.206^{\mathrm{b}}$ & & & $0.016^{\mathrm{b}}$ \\
\hline No & 89.9 & 81.8 & 96.5 & 88.1 & & 82.4 & 97.9 & \\
\hline Yes & 10.1 & 18.2 & 3.5 & 11.9 & & 17.6 & 2.1 & \\
\hline $\begin{array}{c}\text { Maximum weight } \\
\text { usually carried }\end{array}$ & & & & & $0.393^{b}$ & & & $<0.001^{\mathrm{b}}$ \\
\hline Does not carry weight & 61.7 & 81.8 & 69.0 & 54.2 & & 45.0 & 79.2 & \\
\hline Up to $49 \mathrm{Kg}$ & 24.2 & 18.2 & 17.2 & 28.8 & & 27.5 & 20.8 & \\
\hline $50 \mathrm{Kg}$ or over & 14.1 & 0 & 13.8 & 17.0 & & 27.5 & 0 & \\
\hline No & 21.2 & 72.7 & 13.8 & 15.3 & & 17.6 & 25.0 & \\
\hline Yes & 78.8 & 27.3 & 86.2 & 84.7 & & 82.4 & 75.0 & \\
\hline $\begin{array}{l}\text { Wearing protective clothing } \\
\text { during harvest }\end{array}$ & & & & & $0.102^{b}$ & & & $0.812^{\mathrm{a}}$ \\
\hline No & 61.6 & 90.9 & 55.2 & 59.3 & & 62.8 & 60.4 & \\
\hline Yes & 38.4 & 9.1 & 44.8 & 40.7 & & 37.2 & 39.6 & \\
\hline $\begin{array}{l}\text { Wearing gloves } \\
\text { during harvest }\end{array}$ & & & & & $0.699^{\mathrm{b}}$ & & & $0.088^{a}$ \\
\hline No & 50.5 & 63.6 & 48.3 & 49.1 & & 58.8 & 41.7 & \\
\hline Yes & 49.5 & 36.4 & 51.7 & 50.9 & & 41.2 & 58.3 & \\
\hline
\end{tabular}




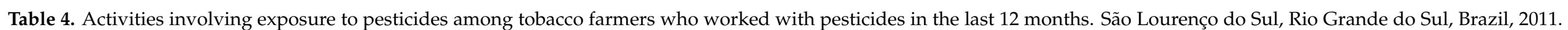
$(n=99)$.

\begin{tabular}{|c|c|c|c|c|c|c|c|c|}
\hline \multirow{3}{*}{ Variables } & \multirow{3}{*}{$\begin{array}{c}\text { Total }(n=99) \\
\%\end{array}$} & \multicolumn{4}{|c|}{ Age Group } & \multicolumn{3}{|c|}{ Sex } \\
\hline & & $<14$ Years $(n=11)$ & $14-15$ Years $(n=29)$ & $16-17$ Years $(n=59)$ & \multirow{2}{*}{$p$-Value for Age } & Male $(n=51)$ & Female $(n=48)$ & \multirow{2}{*}{$p$-Value for Sex } \\
\hline & & $\%$ & $\%$ & $\%$ & & $\%$ & $\%$ & \\
\hline Preparing mixture * & & & & & $0.080^{b}$ & & & $0.013^{b}$ \\
\hline Does not work with pesticide & 50.0 & 81.8 & 48.3 & 44.8 & & 42.0 & 58.3 & \\
\hline Does not prepare & 38.8 & 18.2 & 48.3 & 38.0 & & 38.0 & 39.6 & \\
\hline Prepares & 11.2 & 0 & 3.4 & 17.2 & & 20.0 & 2.1 & \\
\hline Applying* & & & & & $0.059^{\mathrm{b}}$ & & & $0.043^{b}$ \\
\hline Does not work with pesticide & 50.0 & 81.8 & 48.3 & 44.8 & & 42.0 & 58.3 & \\
\hline Does not apply & 37.8 & 18.2 & 48.3 & 36.2 & & 38.0 & 37.5 & \\
\hline Applies & 12.2 & 0 & 3.4 & 19.0 & & 20.0 & 4.2 & \\
\hline Cleaning equipment used to apply * & & & & & $0.112^{\mathrm{b}}$ & & & $0.040^{\mathrm{b}}$ \\
\hline Does not work with pesticide & 50.0 & 81.8 & 48.3 & 44.8 & & 42.0 & 58.3 & \\
\hline Does not clean & 44.9 & 18.2 & 51.7 & 46.6 & & 48.0 & 41.7 & \\
\hline Cleans & 5.1 & 0 & 0 & 8.6 & & 10.0 & 0 & \\
\hline Washing clothes contaminated & & & & & $0.123^{b}$ & & & $0.010^{\mathrm{b}}$ \\
\hline Does not work with pesticide & 50.0 & 81.8 & 48.3 & 44.8 & & 42.0 & 58.3 & \\
\hline Does not wash & 42.9 & 18.2 & 37.9 & 50.0 & & 56.0 & 29.2 & \\
\hline Washes & 7.1 & 0 & 13.8 & 5.2 & & 2.0 & 12.5 & \\
\hline Re-entering the treated field * & & & & & $0.281^{b}$ & & & $0.261^{b}$ \\
\hline Does not work with pesticide & 50.0 & 81.8 & 48.3 & 44.8 & & 42.0 & 58.3 & \\
\hline Goes in & 9.2 & 0 & 6.9 & 12.1 & & 12.0 & 6.3 & \\
\hline
\end{tabular}

Table 5. Health outcomes among tobacco farmers stratified by age group and sex. São Lourenço do Sul, Rio Grande do Sul, Brazil, 2011.

\begin{tabular}{|c|c|c|c|c|c|c|c|c|}
\hline \multirow{3}{*}{ Variables } & \multirow{3}{*}{$\begin{array}{c}\text { Total }(n=12) \\
\%\end{array}$} & \multicolumn{4}{|c|}{ Age Group } & \multicolumn{3}{|c|}{ Sex } \\
\hline & & $<14$ Years $(n=0)$ & $14-15$ Years $(n=1)$ & $16-17$ Years $(n=11)$ & \multirow{2}{*}{$p$-Value for Age } & Male $(n=6)$ & Female $(n=6)$ & \multirow{2}{*}{$p$-Value for Sex } \\
\hline & & $\%$ & $\%$ & $\%$ & & $\%$ & $\%$ & \\
\hline Urinary cotinine $* *$ & & & & & $0.500^{b}$ & & & $0.286^{\mathrm{b}}$ \\
\hline$<20 \mathrm{ng} / \mathrm{mL}$ & 16.7 & - & 0 & 18.2 & & 0 & 33.3 & \\
\hline $20-100 \mathrm{ng} / \mathrm{mL}$ & 33.3 & - & 100 & 27.3 & & 50.0 & 16.7 & \\
\hline$>100 \mathrm{ng} / \mathrm{mL}$ & 50.0 & - & 0 & 54.5 & & 50.0 & 50.0 & \\
\hline
\end{tabular}


Table 5. Cont.

\begin{tabular}{|c|c|c|c|c|c|c|c|c|}
\hline \multirow{3}{*}{ Variables } & \multirow{3}{*}{$\begin{array}{c}\text { Total }(n=99) \\
\%\end{array}$} & \multicolumn{4}{|c|}{ Age Group } & \multicolumn{3}{|c|}{ Sex } \\
\hline & & $<14$ Years $(n=11)$ & 14-15 Years $(n=29)$ & $16-17$ Years $(n=59)$ & \multirow{2}{*}{$p$-Value for Age } & Male $(n=51)$ & Female $(n=48)$ & \multirow{2}{*}{$p$-Value for Sex } \\
\hline & & $\%$ & $\%$ & $\%$ & & $\%$ & $\%$ & \\
\hline GTS in lifetime * & & & & & $0.501^{b}$ & & & $0.197^{b}$ \\
\hline Never & 75.5 & 72.7 & 85.7 & 71.1 & & 82.4 & 68.0 & \\
\hline $1-2$ times & 11.2 & 18.2 & 3.6 & 13.6 & & 9.8 & 12.8 & \\
\hline 3 times or more & 13.3 & 9.1 & 10.7 & 15.3 & & 7.8 & 19.2 & \\
\hline Pesticide poisoning in lifetime & & & & & $1.000^{\mathrm{b}}$ & & & $0.243^{\mathrm{b}}$ \\
\hline No & 97.0 & 100 & 96.5 & 96.6 & & 94.1 & 100 & \\
\hline Coughing without having a cold & & & & & $0.048^{\mathrm{b}}$ & & & $0.679^{a}$ \\
\hline No & 83.8 & 72.7 & 96.5 & 79.7 & & 82.4 & 85.4 & \\
\hline Yes & 16.2 & 27.3 & 3.5 & 20.3 & & 17.6 & 14.6 & \\
\hline Wheezing in the last 12 months & & & & & $0.835^{\mathrm{b}}$ & & & $0.105^{\mathrm{b}}$ \\
\hline No & 93.9 & 90.9 & 96.5 & 93.2 & & 98.0 & 89.6 & \\
\hline Yes & 6.1 & 9.1 & 3.5 & 6.8 & & 2.0 & 10.4 & \\
\hline Neck pain in the last year & & & & & $0.324^{b}$ & & & $0.110^{\mathrm{b}}$ \\
\hline No & 97.0 & 90.9 & 100 & 96.6 & & 100 & 93.7 & \\
\hline Yes & 3.0 & 9.1 & 0 & 3.4 & & 0 & 6.3 & \\
\hline Thoracic spine pain in the last year & & & & & $0.396^{\mathrm{b}}$ & & & $0.524^{\mathrm{a}}$ \\
\hline Low back pain in the last year & & & & & $0.041^{\mathrm{b}}$ & & & $0.979^{\text {a }}$ \\
\hline No & 70.7 & 81.8 & 86.2 & 61.0 & & 70.6 & 70.8 & \\
\hline Yes & 29.3 & 18.2 & 13.8 & 39.0 & & 29.4 & 29.2 & \\
\hline
\end{tabular}




\section{Discussion}

Child labor in tobacco farming is prohibited for anyone under 18 years old [3]. Notwithstanding, in 79 of the 912 properties studied, mostly small and medium-sized family farms, there were young people under 18 years old who had worked in the year prior to the interview. The majority started working when they were less than 15 years old, for more than $5 \mathrm{~h}$ a day, increasing the time they spent working during the harvest period. There was also a high prevalence of age-grade mismatches among 16 and 17 years old. The activities which these young people carried out most, tying hands of tobacco and picking, occurred during the harvest, which coincides with school holidays. In addition, the young people had performed a variety of activities both during the harvest and during the rest of the year, including some high-risk activities such as working with pesticides, driving tractors, and cutting trees with chainsaws.

A study conducted by Arcury et al. (2016) [36] indicated that tobacco farmers had much higher levels of urinary cotinine $(396.03 \mathrm{ng} / \mathrm{mL})$ when compared to individuals who did not work in tobacco farming $(9.03 \mathrm{ng} / \mathrm{mL})$. Exposure to green tobacco leaves causes transdermal absorption of nicotine, thus increasing nicotine levels in the blood and resulting in GTS. Nicotine is water-soluble [36], and as such, its absorption increases when harvesting leaves are wetted by morning dew or due to contact with transpiration. Absorption is also increased by not using personal protective equipment, by eventual impairment of the skin integrity, and vasodilation resulting from working in hot and humid environments [37-41].

In the present study, the strong participation of young people in tying hands of tobacco and harvesting, with significant exposure to nicotine, is reflected in the percentage of those who had detectable urinary cotinine and had already had GTS. There are indications that workers who are more exposed and who are smokers can develop nicotine tolerance. Thus, younger workers [19], children and adolescents [42], individuals with less working time [32,42], and nonsmokers [19,32,42] are at greater risk of developing GTS. The consequences of massive nicotine exposure in the medium and long term are still little studied. Within the same study, when analyzing tobacco growers 18 years or older, a positive linear association of the number of GTS episodes with mental disorders and with respiratory symptoms was confirmed $[14,20]$.

Studies with farmers indicate that exposure to pesticides has both acute effects, such as poisoning, as well as long-term effects. The effects of pesticides on health vary according to the type of chemical used, and multi-chemical exposure makes them more complex to assess. The most studied pesticides are organophosphates, which are neurotoxic and have been associated with mental health problems such as depression [14,16,43-45] and cognitive deficits [46]. Other analyses within the overall scope of this study indicated that pesticide poisoning doubled the risk of suicidal ideation [47]. Children and adolescents are probably more susceptible to chemical exposure since they are in a period of maturation of their endocrine system, and such exposure could alter the delicate hormonal balance and their biofeedback mechanisms [24,25]. In the present study, children over 13 years old carried out several activities involving pesticide exposure and reported episodes of acute pesticide poisoning. Thus, the potential long-term health effects of pesticides on children and adolescents are of concern.

Tying hands of tobacco is one of the main activities undertaken by young people in tobacco farming. In general, it is done inside the barn so that workers are exposed to organic dust that increases the risk of respiratory problems [48]. A North American study [21] with children who worked in agriculture found that $16.4 \%$ had wheezing at some time in their lives, $14.3 \%$ had a persistent cough for more than ten days, and one-third had two or more respiratory problems. The prevalence of coughing without having a cold in the present study was higher than in the North American study [21] and, considering the difference in recall, $6.1 \%$ prevalence of wheezing in the last year was considerable. Association between respiratory problems and contact with dust, pesticides, and microorganisms is well established [15,49-52]. There is evidence that inhaling nicotine is associated with 
respiratory problems $[53,54]$; thus, the nicotine in tobacco leaf dust may increase the risk of this type of problem. However, there are no studies that assess whether the high dermal absorption of nicotine also potentiates the risk of respiratory problems [20].

Reis et al. (2017) [10] emphasize that tobacco growing in family farming requires manual work and often exposes workers to repetitive movements, excessive weightlifting, and inappropriate postures. Given the activities performed, work-related musculoskeletal disorders in the shoulder, elbow, knee, and hip, as well as in the different segments of the spine, become frequent and are evidenced by several studies with farmers of different ages [9,11-13]. Harvesting and tying hands of tobacco involves repetitive movements and bending and sitting postures. Other activities such as carrying weight (predominantly done by boys) and lifting tobacco sticks also place heavy demands on the musculoskeletal system because apart from the posture and movement of the upper limbs, they demand physical strength.

Quandt et al. (2021) [13] found that $6.4 \%$ of young people performing farm work reported low back pain in at least one site during physical examination. In the present study, more than one-third of young people aged 16-17 reported low back pain in the last year. This may be related to greater involvement with work and the fact that they have been working longer when compared with younger children. There is a concern that children and adolescents are more vulnerable than adults to ergonomic exposures since, as they are in a period of rapid growth, they could be at increased risk of injuries to bone epiphyses or ligaments. In addition, the long-term effect of ergonomic exposure on musculoskeletal disorders is unclear [24,25].

It is important to consider that these aspects are aggravated by long working hours typical of the harvest period. Quandt et al. (2021) [13] indicate that children involved in agricultural work perform less intense tasks and for less time than adults, corroborating what was found in the present study, namely that children and adolescents worked for fewer hours outside the harvest period and that their involvement in different farming tasks increased with age. However, during the harvest, most adolescents work in a similar way to adults, performing diversified tasks and working more than seven hours a day.

Among the limitations of the present study, it is important to recognize the occurrence of selection bias due to the fact that working in the tobacco industry is prohibited for people under 18 years old [3]. This certainly caused child labor to be partly hidden, preventing the identification of young workers by the study. The illegal nature of this work may also have caused information bias, underestimating work in activities of higher risk such as exposure to pesticides and driving a tractor, among others. On the other hand, it is possible that the young people identified are those who participate more strongly in production activities. The urinary cotinine samples were obtained just from 12 young workers, and pesticide poisoning was not evaluated through biomarkers but rather characterized by self-reported information. These aspects limited the chemical exposure and related outcomes evaluation.

Data collection for this study took place in 2011. Since then, there have been no considerable changes in the production system that could substantially alter tobacco farming. However, there were contextual variations. Considering the reduction in tobacco consumption and the production migration to low-income countries increasing competition, policies were implemented to encourage crop diversification. In addition, the COVID-19 pandemic has caused schools to suspend in-person activities and may have led tobacco producers to avoid hiring temporary workers. These aspects can modify both the prevalence of child labor and the type of activity that children carry out in tobacco production.

\section{Conclusions}

Despite the prohibition, this study pointed out the persistence of young people under 18 years old working in tobacco growing, in various activities, the most frequent being harvesting tobacco leaves and tying hands of tobacco. Chemical exposure to nicotine and pesticides, ergonomic exposure, such as working in awkward positions, and physical expo- 
sure, such as exposure to dust, resulted in a high prevalence of GTS, pesticide poisoning, respiratory, and musculoskeletal problems.

Future studies on child and adolescent populations in relation to tobacco growing are needed to assess the health of workers and nonworkers with larger sample sizes. Nonworkers may also be exposed to some extent by being with their parents on the plantation or in the barn. In addition, studies should intensify objective assessment of exposure to dust, nicotine, and pesticide, as well as assessment of outcomes such as pesticide poisoning and lung function.

Considering that working in tobacco growing takes place in the context of family farming, it is fundamental to provide clarification to both adults and young people about the forms of exposure present in this type of work and its effect on health. Health workers are privileged actors and should be trained to identify and report situations of child labor in tobacco farming. They should also be prepared to recognize GTS, pesticide poisoning, as well as respiratory and musculoskeletal problems and relate them to this form of work, providing relevant guidance for rehabilitation and prevention of new episodes. The implementation of full-time agricultural schools can provide knowledge about sustainable agricultural production models, reducing the rates of age-grade mismatch without taking young people away from rural areas.

Supplementary Materials: The following are available online at https: / www.mdpi.com/article / 10.3390 /ijerph182212255/s1, File S1: Individual Questionnaire—Selected Questions—Characterization of Health Problems.

Author Contributions: Conceptualization, A.G.F., N.M.X.F., R.D.M. and N.S.F.; data curation, R.D.M. and N.S.F.; Formal analysis, A.G.F., N.M.X.F., A.L.S.C.S. and M.P.d.C.; funding acquisition, A.G.F., N.M.X.F. and R.D.M.; investigation, A.G.F., N.M.X.F., R.D.M. and N.S.F.; methodology, A.G.F., N.M.X.F., R.D.M. and N.S.F.; project administration, A.G.F.; supervision, A.G.F., N.M.X.F., R.D.M. and N.S.F.; visualization, A.G.F., N.M.X.F., A.L.S.C.S., R.D.M., N.S.F. and M.P.d.C.; writingoriginal draft, A.G.F., N.M.X.F., A.L.S.C.S. and M.P.d.C.; writing-review \& editing, A.G.F., N.M.X.F., A.L.S.C.S., R.D.M., N.S.F. and M.P.d.C. All authors have read and agreed to the published version of the manuscript.

Funding: This research was funded by the National Council for Scientific and Technological Development (CNPq Process 483214/2009-4, 2009) and by the Research Support Foundation of the Rio Grande do Sul State (FAPERGS Process 09/0057.5, 2009).

Institutional Review Board Statement: The study was conducted according to the guidelines of the Declaration of Helsinki, and approved by the Research Ethics Committee of the Faculty of Medicine of the Federal University of Pelotas (protocol code 11/2010).

Informed Consent Statement: Informed consent was obtained from all young people involved in the study and one of their legal guardians.

Data Availability Statement: The data presented in this study are not publicly available due to ethical reasons. Data are available from the corresponding author upon reasonable request.

Conflicts of Interest: The authors declare no conflict of interest.

\section{References}

1. Santos, C.E. Anuário Brasileiro do Tabaco. Santa Cruz Do Sul: Editora Gazeta Santa Cruz. 2020. Available online: https://www. editoragazeta.com.br/sitewp/wp-content/uploads/2021/01/TABACO_2020_DUPLAS.pdf (accessed on 14 September 2021).

2. International Labour Organization-ILO. C182-Convenção Sobre Proibição Das Piores Formas De Trabalho Infantil E Ação, 2000 Imediata Para Sua Eliminação. Available online: https://www.ilo.org/brasilia/convencoes/WCMS_236696/lang--pt/index.htm (accessed on 11 July 2021).

3. Brasil. Decreto 6.481 De 12 De Junho De 2008. Regulamenta Os Artigos $3^{\circ}$, Alínea “D”, E $4^{\circ}$ da Convenção 182 da Organização Internacional Do Trabalho (OIT) Que Trata da Proibição Das Piores Formas De Trabalho Infantil E Ação Imediata Para Sua Eliminação, Aprovada Pelo Decreto Legislativo N 178, De 14 De Dezembro De 1999, E Promulgada Pelo Decreto $n^{\circ} 3.597$, De 12 De Setembro De 2000, E Dá Outras Providências. Available online: www.planalto.gov.br/ccivil_03/_ato2007-2010/2008/decreto/ d6481.htm (accessed on 11 July 2021). 
4. International Labour Organization-ILO. Trabalho Infantil. Available online: https://www.ilo.org/brasilia/temas/trabalhoinfantil/lang--pt/index.htm (accessed on 11 July 2021).

5. Custódio, A.V.; Cabral, M.E.L. Trabalho infantil na agricultura familiar: Uma violação de direitos humanos perpetuada no meio rural. Rev. Jurídica Pauta 2019, 1, 3-15.

6. Parker, D. Health effect on child labour. Lancet 1997, 350, 1395-1396. [CrossRef]

7. Instituto Brasileiro de Geografia e Estatística-IBGE. Pesquisa Nacional Por Amostra De Domicílios Contínua-Trabalho De Crianças E Adolescentes De 5 a 17 Anos De Idade 2016-2019. 2020. Available online: https:/ / biblioteca.ibge.gov.br/visualizacao/ livros/liv101777_informativo.pdf (accessed on 11 July 2021).

8. Instituto Brasileiro de Geografia e Estatística-IBGE. Censo Agro 2017. Available online: https:/ / censos.ibge.gov.br/2013-agenciade-noticias / releases / 18383-pnad-continua-2016-brasil-tem-pelo-menos-998-mil-criancas-trabalhando-em-desacordo-com-alegislacao.html (accessed on 11 July 2021).

9. Meucci, R.D.; Fassa, A.G.; Faria, N.M.X.; Fiori, N.S. Chronic low back pain among tobacco farmers in southern Brazil. Int. J. Occup. Environ. Health 2015, 21, 66-73. [CrossRef] [PubMed]

10. Reis, M.M.; Oliveira, A.P.N.; Turci, S.R.B.; Dantas, R.M.; Silva, V.S.P.; Gross, C.; Jensen, T.; Costa e Silva, V.L. Conhecimentos, atitudes e práticas de agricultoras sobre o processo de produção de tabaco em um município da Região Sul do Brasil. Cad. Saúde Pública 2017, 33 (Suppl. S3), S148-S161. [CrossRef]

11. Fassa, A.G.; Fiori, N.S.; Meucci, R.D.; Faria, N.M.X.; Carvalho, M.P. Neck pain among tobacco farm workers in Southern Brazil. Salud Colect. 2020, 16, e2307. [CrossRef]

12. Carvalho, M.P.; Fiori, N.S.; Meucci, R.D.; Faria, N.M.X.; Fassa, A.G. Thoracic spine pain and associated factors among tobacco farmers. Rev. Bras. Saúde Ocup. 2020, 45, e33. [CrossRef]

13. Quandt, S.A.; Arnold, T.J.; Talton, J.W.; Miles, C.M.; Mora, D.C.; Arcury, T.A.; Daniel, S.S. Musculoskeletal injury symptoms among hired Latinx child farmworkers in North Carolina. Am. J. Ind. Med. 2021, 64, 620-628. [CrossRef]

14. Faria, N.M.X.; Fassa, A.G.; Meucci, R.D.; Fiori, N.S.; Miranda, V.I. Occupational exposure to pesticides, nicotine and minor psychiatric disorders among tobacco farmers in southern Brazil. Neurotoxicology 2014, 45, 347-354. [CrossRef]

15. Faria, N.M.X.; Facchini, L.A.; Fassa, A.G.; Tomasi, E. Trabalho rural, exposição a poeiras e sintomas respiratórios entre agricultores. Rev. Saúde Pública 2006, 40, 827-836. [CrossRef]

16. London, L.; Beseler, C.; Bouchard, M.F.; Bellinger, D.C.; Colosio, C.; Grandjean, P.; Harari, R.; Kootbodien, T.; Kromhout, H.; Little, F.; et al. Neurobehavioral and neurodevelopmental effects of pesticides exposures. Neurotoxicology 2012, 33, 887-896. [CrossRef]

17. Fassa, A.G.; Meucci, R.D.; Fiori, N.S.; Carrett, M.L.V.; Faria, N.M.X. Urinary cotinine in tobacco farmers in Southern Brazil. Rev. Saúde Pública 2018, 52, 70. [CrossRef]

18. Ballard, T.; Ehlers, J.; Freund, E.; Auslander, M.; Brandt, V.; Halperin, W. Green tobacco sickness: Occupational nicotine poisoning in tobacco workers. Arch. Environ. Health 1995, 50, 384-389. [CrossRef]

19. Fassa, A.G.; Faria, N.M.; Meucci, R.D.; Fiori, N.S.; Miranda, V.I.; Facchini, L.A. Green Tobacco Sickness among Tobacco Farmers in Southern Brazil. Am. J. Ind. Med. 2014, 57, 726-735. [CrossRef]

20. Fiori, N.S.; Fassa, A.G.; Faria, N.M.X.; Meucci, R.D.; Miranda, V.I.; Christiani, D.C. Wheezing in tobacco farm workers in southern Brazil. Am. J. Ind. Med. 2015, 58, 1217-1228. [CrossRef]

21. Kearney, G.D.; Arcury, T.A.; Quandt, S.A.; Talton, J.W.; Arnold, T.J.; Sandberg, J.C.; Wiggins, M.F.; Daniel, S.S. Respiratory Health and Suspected Asthma among Hired Latinx Child Farmworkers in Rural North Carolina. Int. J. Environ. Res. Public Health 2020, 17, 7939. [CrossRef]

22. Corriols, M.; Aragón, A. Child labor and acute pesticide poisoning in Nicaragua: Failure to comply with children's rights. Int. J. Occup. Environ. Health 2010, 16, 193-200. [CrossRef]

23. Meucci, R.D.; Fassa, A.G.; Faria, N.M.X.; Fiori, N.S.; Miranda, V.I.; Resende, D. Limitação no trabalho por dor lombar em fumicultores do sul do Brasil. Rev. Bras. Saúde Ocup. 2014, 39, 6-16. [CrossRef]

24. Fassa, A.G.; Facchini, L.A.; Dall'Agnol, M.M.; Christiani, D.C. Child labor and health: Problems and perspectives. Int. J. Occup. Environ. Health 2000, 6, 55-62. [CrossRef]

25. National Research Council and Institute of Medicine. Committee on the Health and Safety Implications of Child Labor. Protecting Youth at Work: Health, Safety, and Development of Working Children and Adolescents in the United States. 1998. Available online: https:/ / www.nap.edu/catalog/6019/protecting-youth-at-work-health-safety-and-development-of-working (accessed on 11 July 2021).

26. Fundo das Nações Unidas para a Infância-UNICEF. Panorama da Distorção Idade-Série No Brasil. 2018. Available online: https: //www.unicef.org/brazil/media/461/file/Panorama_da_distorcao_idade-serie_no_Brasil.pdf (accessed on 13 August 2021).

27. Organização Mundial da Saúde. Estratégias de Autoajuda para Diminuir ou Parar o Uso De Substâncias: Um Guia; FIOCRUZ/INI: Rio de Janeiro, Brasil, 2020.

28. Kuorinka, I.; Jonsson, B.; Kilbom, A.; Vinterberg, H.; Biering-Sørensen, F.; Andersson, G.; Jørgensen, K. Standardised Nordic questionnaires for the analysis of musculoskeletal symptoms. Appl. Ergon. 1987, 18, 233-237. [CrossRef]

29. Pinheiro, F.A.; Trócolli, B.T.; Carvalho, C.V. Validação do questionário nórdico de sintomas osteomusculares como medida de morbidade. Rev. Saúde Pública 2002, 36, 307-312. [CrossRef] 
30. Meucci, R.D.; Fassa, A.G.; Paniz, V.M.V.; Silva, M.C.; Wegman, D.H. Increase of chronic low back pain prevalence in a mediumsized city of southern Brazil. BMC Musculoskelet Disord. 2013, 14, 155. [CrossRef]

31. Carvalho, M.P.; Schmidt, L.G.; Soares, M.C.F. Musculoskeletal disorders and their influence on the quality of life of the dockworker: A cross-sectional study. Work 2016, 53, 805-812. [CrossRef]

32. Arcury, T.A.; Vallejos, Q.M.; Schulz, M.R.; Feldman, S.R.; Fleischer, A.B., Jr.; Verma, A.; Quandt, S.A. Green tobacco sickness and skin integrity among migrant Latino farmworkers. Am. J. Ind. Med. 2008, 51, 195-203. [CrossRef]

33. Benowitz, N.L.; Nardone, N.; Hatsukami, D.K.; Donny, E.C. Biochemical estimation of noncompliance with smoking of very low nicotine content cigarettes. Cancer Epidemiol. Biomarkers Prev. 2015, 24, 331-335. [CrossRef]

34. Petersen, G.O.; Leite, C.E.; Chatkin, J.M.; Thiesen, F.V. Cotinine as a biomarker of tobacco exposure: Development of a HPLC method and comparison of matrices. J. Sep. Sci. 2010, 33, 516-521. [CrossRef]

35. Cattaneo, R.; Alegretti, A.P.; Sagebin, F.R.; Abreu, C.M.; Petersen, G.O.; Chatkin, J.M. Validação do método para determinação de cotinina em urina por cromatografia líquida de alta eficiência. Rev. Bras. Toxicol. 2006, 19, 25-31.

36. Arcury, T.A.; Laurienti, P.J.; Talton, J.W.; Chen, H.; Howard, T.D.; Summers, P.; Quandt, S.A. Urinary Cotinine Levels among Latino Tobacco Farmworkers in North Carolina Compared to Latinos Not Employed in Agriculture. Nicotine Tob. Res. 2016, 18, 1517-1525. [CrossRef]

37. Arcury, T.A.; Quandt, S.A.; Preisser, J.S. Measuring occupational illness incidence and prevalence in a difficult to study population: Green tobacco sickness among Latino farmworkers in North Carolina. J. Epidemiol. Community Health 2001, 55, 818-824. [CrossRef]

38. Quandt, S.A.; Arcury, T.A.; Preisser, J.S.; Norton, D.; Austin, C. Migrant farmworkers and green tobacco sickness: New issues for an understudied disease. Am. J. Ind. Med. 2000, 37, 307-315. [CrossRef]

39. Benowitz, N.L.; Jacob III, P.; Olsson, P.; Johansson, C.J. Intravenous nicotine retards transdermal absorption of nicotine: Evidence of blood flow-limited percutaneous absorption. Clin. Pharmacol. Ther. 1992, 52, 223-230. [CrossRef]

40. Benowitz, N.L.; Zevin, S.; Jacob III, P. Sources of variability in nicotine and cotinine levels with use of nicotine nasal spray, transdermal nicotine, and cigarette smoking. Br. J. Clin. Pharmacol. 1997, 43, 259-267. [CrossRef] [PubMed]

41. Ghosh, S.K.; Gokani, V.N.; Parikh, J.R.; Doctor, P.B.; Kashyap, S.K.; Chatterjee, B.B. Protection against "green symptoms" from tobacco in Indian harvesters: A preliminary intervention study. Arch. Environ. Health 1987, 42, 121-124. [CrossRef] [PubMed]

42. Centers for Disease Control and Prevention-The National Institute for Occupational Safety and Health (NIOSH). Recommended Practices: Green Tobacco Sickness. Available online: https:/ /www.cdc.gov/niosh/docs/2015-104/default.html (accessed on 4 September 2021).

43. London, L.; Flisher, A.J.; Wesseling, C.; Mergler, D.; Kromhout, H. Suicide and exposure to organophosphate insecticides: Cause or effect? Am. J. Indus. Med. 2005, 47, 308-321. [CrossRef] [PubMed]

44. Lima, C.S.; Nunes-Freitas, A.L.; Ribeiro-Carvalho, A.; Filgueiras, C.C.; Manhães, A.C.; Meyer, A.; Abreu-Villaça, Y. Exposure to methamidophos at adulthood adversely affects serotonergic biomarkers in the mouse brain. Neurotoxicology 2011, 32, 718-724. [CrossRef] [PubMed]

45. Keifer, M.C.; Firestone, J. Neurotoxicity of pesticides. J. Agromed. 2007, 12, 17-25. [CrossRef]

46. Rasoul, G.M.A.; Salem, M.E.A.; Mechael, A.A.; Hendy, O.M.; Rohlman, D.S.; Ismail, A.A. Effects of occupational pesticide exposure on children applying pesticides. Neurotoxicology 2008, 29, 833-838. [CrossRef]

47. Szortyka, A.L.C.; Faria, N.M.X.; Carvalho, M.P.; Feijó, F.R.; Meucci, R.D.; Flesch, B.D.; Fiori, N.S.; Fassa, A.G. Suicidality among South Brazilian tobacco growers. Neurotoxicology 2021, 86, 52-58. [CrossRef]

48. Zergham, A.; Heller, D. Farmers Lung. In StatPearls [Internet]; StatPearls Publishing: Treasure Island, FL, USA, 2021. Available online: https:/ / pubmed.ncbi.nlm.nih.gov/32491512/ (accessed on 10 September 2021).

49. American Thoracic Society. Medical Section of the American Lung Association. Respiratory Health Hazards in Agriculture. Am. J. Respir. Crit. Care Med. 1998, 158, S1-S76. [CrossRef]

50. Villarejo, D.; Baron, S.L. The occupational health status of hired farm workers. Occup. Med. 1999, 14, $613-635$.

51. Karvonen, A.M.; Hyvärinen, A.; Gehring, U.; Korppi, M.; Doekes, G.; Riedler, J.; Braum-Fahrländer, C.; Bitter, S.; Schmid, S.; Keski-Nisula, L.; et al. Exposure to microbial agents in house dust and wheezing, atopic dermatitis and atopic sensitization in early childhood: A birth cohort study in rural areas. Clin. Exp. Allergy 2012, 42, 1246-1256. [CrossRef]

52. May, S.; Romberger, D.J.; Poole, J.A. Respiratory health effects of large animal farming environments. J. Toxicol. Environ. Health B. Crit. Rev. 2012, 15, 524-541. [CrossRef]

53. Ahmad, S.; Zafar, I.; Mariappan, N.; Husain, M.; Wei, C.; Vetal, N.; Eltoum, I.A.; Ahmad, A. Acute pulmonary effects of aerosolized nicotine. Am. J. Physiol. Lung Cell Mol. Physiol. 2019, 316, L94-L104. [CrossRef]

54. Barna, S.; Rózsad, D.; Vargab, J.; Fodorc, A.; Szilasic, M.; Galuskab, L.; Garaia, I. First comparative results about the direct effect of traditional cigarette and e-cigarette smoking on lung alveolocapillary membrane using dynamic ventilation scintigraphy. Nucl. Med. Commun. 2019, 40, 153-158. [CrossRef] 